\title{
Repères bibliographiques du dossier "L'enseignement des langues en Europe : enjeux et stratégies »
}

\section{Marie-France Pamart}

\section{OpenEdition}

\section{Journals}

Édition électronique

URL : http://journals.openedition.org/ries/1758

DOI : $10.4000 /$ ries. 1758

ISSN : 2261-4265

Éditeur

Centre international d'études pédagogiques

Édition imprimée

Date de publication : 1 septembre 2003

Pagination : 117-128

ISBN : 978-2-85420-557-1

ISSN : 1254-4590

\section{Référence électronique}

Marie-France Pamart, «Repères bibliographiques du dossier « L'enseignement des langues en

Europe : enjeux et stratégies » », Revue internationale d'éducation de Sèvres [En ligne], 33 | septembre 2003, mis en ligne le 23 novembre 2011, consulté le 01 mai 2019. URL : http://

journals.openedition.org/ries/1758; DOI : 10.4000/ries.1758 


\title{
Repères bibliographiques
}

\section{Marie-France Pamart}

Cette bibliographie sélective est axée sur les politiques linguistiques; elle ne comporte donc pas de références ayant trait à la didactique, aux méthodes d'enseignement ou à la formation des enseignants. Les documents cités, postérieurs à 1996, sont consultables au CIEP ou en ligne; leurs notices sont organisées en quatre parties: ouvrages de références, études de la Commission européenne, études du Conseil de l'Europe, études consacrées à l'Année européenne des langues 2001

\section{Ouvrages de références}

\author{
BAGGIONI Daniel, Langues et nations en Europe, Paris, Payot (Bibliothèque \\ scientifique Payot), 1997, 378 p., bibliogr.
}

L'auteur est arrivé à la conviction que les processus de formation d'une langue nationale ont partie liée avec les processus de construction nationale. Après avoir brossé la situation des langues dans l'Europe d'aujourd'hui et avoir posé les concepts nécessaires à une bonne entente interdisciplinaire, il distingue les époques suivantes: la première révolution écolinguistique en Europe occidentale (XV $\mathrm{XVII}^{\mathrm{e}}$ siècles), l'Europe des langues communes (1550-1880), la deuxième révolution écolinguistique en Europe (1880-1918), le temps des nations révolutionnaires et romantiques (XIX ${ }^{\mathrm{e}}$ siècle), l'achèvement du processus de partage de l'Europe des nations. Il termine sur un chapitre intitulé «Vers une troisième révolution écolinguistique en Europe» avant de conclure sur la communication entre langues nationales et internationales dans l'Europe de demain.

BOLLMANN Yvonne, La bataille des langues en Europe: essai, Paris, Bartillat, 2001, 174 p., bibliogr.

En 1992, le Conseil de l'Europe a adopté la Charte des langues régionales et minoritaires, qui a été l'occasion de nombreux débats en France. L'auteur revient sur les origines et les objectifs de ce texte, en s'appuyant sur une documentation puisée en Allemagne, en Autriche et en France. Elle explicite notamment la notion de minorité évoquée dans la Charte et porte un regard critique sur l'imbrication entre culture et politique.

CALVET Louis-Jean, Le marché aux langues : essai de politologie linguistique sur la mondialisation, Paris, Plon, 2002, 220 p., bibliogr., annexes.

L'auteur dresse un bilan linguistique du monde, en associant théorie et études de cas. Il pose les principes d'une science des pratiques en politiques linguistiques, la politologie linguistique. La première partie présente les attentes de la politologie linguistique et ses instruments. La seconde analyse le discours "politico-linguistique correct», à l'aide d'exemples concrets issus de l'étude synchronique et diachronique des langues. La troisième examine le versant linguistique de la mondialisation. Pour terminer, l'auteur élabore des propositions concrètes de gestion du monde plurilingue.

CALVET Louis-Jean, La guerre des langues et les politiques linguistiques, Paris, Hachette Littératures (Pluriel), 1999, 294 p.

L'auteur, à partir d'enquêtes et d'études de cas concrets, africains, latino-américains, européens et asiatiques, analyse les enjeux de la politique linguistique. Dans une première partie, 
Louis-Jean Calvet pose la question de l'origine des langues et propose quelques hypothèses pour en comprendre la genèse. Les deux dernières parties portent, d'une part, sur l'étude de la guerre des langues in vivo, manifestations au plan linguistique des conflits humains suivi d'une étude sur la planification linguistique.

\section{CALVET Louis-Jean, Les politiques linguistiques, Paris, PUF (Que sais-je ?),} 1996, $127 p$.

L'auteur expose d'abord les origines de la politique linguistique. Il dresse ensuite une typologie des situations plurilingues et décrit les instruments de la planification linguistique. A travers des exemples précis, il montre comment les politiques linguistiques interviennent sur la forme de la langue et sur son statut.

CHAUDENSON Robert éd., L'Europe parlera-t-elle anglais demain ? Actes du Colloque international de Bordeaux organisé par le Goethe Institut et l'INTIF le 3 mars 2001, Paris, L'Harmattan (Langues et développement), 2001, 180 p.

Cet ouvrage est le compte-rendu de la manifestation qui s'est tenue le 3 mars 2001 à Bordeaux, à l'initiative du Goethe Institut, sur le thème «L'Europe parlera-t-elle anglais demain ?». L'année 2001 a été déclarée "Année européenne des langues» par l'Union Européenne et le Conseil de l'Europe. Ce projet avait pour but de célébrer la diversité linguistique et de promouvoir l'apprentissage des langues. L'ouvrage se veut un plaidoyer pour le pluralisme linguistique et contre l'hégémonie de l'anglais.

CENTRE INTERNATIONAL D'ETUDES PEDAGOGIQUES (CIEP). Maison des langues, Principales certifications en langues étrangères: diplômes et tests: guide 2002, Sèvres, France, CIEP, 2002, 36 p.

118 Ce guide présente les principales certifications françaises et étrangères pour les langues étrangères.

DICKSON Peter ed., CUMMING Alister ed., INTERNATIONAL ASSOCIATION FOR THE EVALUATION OF EDUCATIONAL ACHIEVEMENT (IEA), Amsterdam, Profiles of language education in 25 countries: overview of phase 1 of the IEA language education study, Londres, National foundation for educational research, 1996, $134 \mathrm{p}$.

Pour chacun des pays étudiés, on présente, d'après une enquête de 1995, la politique linguistique, la place des langues dans l'enseignement (organisation, programmes, formation des enseignants) et les évolutions dans ce domaine, ainsi qu'une courte bibliographie et les coordonnées d'une personne ressource. En annexe, des tableaux récapitulatifs permettent de comparer, dans ces vingt-cinq pays, la situation de l'enseignement de l'anglais, du français, de l'allemand et de l'espagnol.

GAUDEMAR Jean-Paul de prés., LÜDI Georges, BOYD John, Apprendre des langues étrangères: quelles langues et pour quoi faire?, Paris, ministère de l'Éducation nationale, direction de l'Enseignement scolaire (Les rencontres de la DESCO), mars 2002, 22 .

Après une présentation de la réforme engagée par le ministère de l'Éducation en matière d'enseignement des langues étrangères, la parole est donnée à deux spécialistes étrangers de Grande-Bretagne et de Suisse. Le premier conférencier, Sir John Boyd, livre sa vision de la situation britannique actuelle en présentant une étude sur l'enseignement des langues (rapport Nuffield) qui révèle l'étendue de la crise. Le deuxième décrit la situation d'un pays où le multilinguisme (l'allemand, l'italien, le français et le romanche) a statut officiel et a, de ce fait, une certaine avance en la matière. 
GAUTHIER Guy dir., "Langues: une guerre à mort», Panoramiques, $n^{\circ}$ 48, 2000, 215 p., bibliogr.

Ce numéro dresse un panorama général des langues parlées et des politiques linguistiques de différents pays en établissant des comparaisons entre les langues majoritaires, minoritaires et en donnant une définition du plurilinguisme.

GODARD Roger coord., "Des langues vivantes à l'école: dossier", Revue internationale d'éducation Sèvres, $n^{\circ}$ 9, 1996/03, p. 29-139, bibliogr.

La question de l'enseignement des langues vivantes à l'école élémentaire se pose différemment selon les pays et les contextes socio-culturels. Les articles de ce dossier présentent la situation de cet apprentissage dans différents pays: France, Royaume-Uni, Allemagne, Australie, Luxembourg, Suisse, Québec, Italie. Ils permettent ainsi des comparaisons et des réflexions sur la formation des enseignants, le matériel didactique, la coordination pédagogique, le choix des langues.

\section{GRADDOL David, The future of english, Paris, British Council, 1997, 64 p.}

Cette brochure a été conçue par le British Council pour faciliter un débat ouvert et documenté sur l'utilisation future de l'anglais dans le monde, ainsi que sur son enseignement, tout en voulant mettre le savoir-faire britannique en bonne place dans ce nouvel échiquier. Étude prospective, elle propose une projection sur l'utilisation dans langues dans le monde en 2050. Elle propose des données historiques et actuelles sur la langue anglaise et sa diffusion, des réflexions sur les tendances de la mondialisation domaine par domaine, et sur l'impact de cette expansion sur la langue elle-même.

HERRERAS José Carlos éd., L'enseignement des langues étrangères dans les pays de l'Union européenne, Louvain-La-Neuve/Belgique (Bibliothèque des Cahiers de l'Institut de Linguistique de Louvain), 1998, 401 p.

Certains chapitres de cette publication ont fait l'objet d'une présentation lors du colloque international sur «les langues étrangères dans l'Union européenne», tenu à l'université de Valenciennes les 14 et 15 décembre 1995. L'élargissement de l'Europe et la volonté de maintenir onze langues officielles au sein de l'Union européenne posent un problème linguistique majeur, celui de la communication entre les états (traduction/interprétation). Les contributions permettent de comparer les situations d'apprentissage des langues dans les quinze pays de l'Union: le nombre d'années destinées à l'apprentissage des langues étrangères, le nombre de langues dont l'apprentissage est obligatoire, les possibilités de choix et les connaissances pratiques dans ce domaine.

HERRERAS José Carlos, "De l'apprentissage à la maîtrise des langues étrangères en Europe", Études de linguistique appliquée, $n^{\circ} 123-124$, juillet-décembre 2001, p. 313-323.

La maîtrise des langues étrangères devient une nécessité dans le contexte européen afin qu'une meilleure compréhension entre les peuples soit assurée ainsi qu'une cohésion plus forte au sein de l'Union. Dans cet article, l'auteur analyse la situation des langues étrangères en Europe sous deux angles différents: d'un côté, celui de l'apprentissage des langues et de l'autre, celui de la maîtrise des langues. Malheureusement, le constat est celui d'un échec pour ce qui est la diversification des langues dans les systèmes d'enseignement.

\section{LUGINBÜHL Odile coord., "Langue maternelle, langue d'enseignement", Revue internationale d'éducation Sèvres, $n^{\circ} 19$, septembre 1998, p. 19-94.}

Ce dossier analyse à travers des configurations linguistiques et culturelles variées, les réponses aux questions que pose l'enseignement de la langue première, tant en terme de politique éducative que de didactique. 
MAURAIS Jacques coord., "Géostratégies des langues», Sainte-Foy/Canada, Publications du Québec, Terminogramme, $n^{\circ}$ 99-100, décembre 2001, 405 p.

Ce numéro aborde la question linguistique d'un point de vue géostratégique c'est-à-dire en analysant plus globalement les rapports de force gouvernant les choix linguistiques dans des entités géopolitiques ayant leur identité propre et leurs langues d'usage. L'observation de ces stratégies permet une compréhension plus approfondie des motivations collectives et individuelles qui sous-tendent les choix et les pratiques linguistiques.

OLBERT Jürgen, "L'Europe et l'apprentissage des langues: domination d'un seul idiome ou pluralité linguistique ?», Französisch heute, $n^{\circ}$ 4, décembre 1996, p. 259-271.

L'auteur appelle, dans cette communication, à une Europe linguistique pluraliste, qui ne placerait pas l'anglais en position d'hégémonie, mais favoriserait l'enseignement d'autres langues étrangères.

\section{OUANE Adama, IUE: Institut de l'Unesco pour l'éducation/Hambourg/} Allemagne, Vers une culture multilingue de l'éducation, Paris, L'Harmattan, 1996, XV-472 p.

Les auteurs ont mené, dans seize pays d'Afrique, d'Amérique latine et d'Asie, un travail d'analyse des pratiques langagières qui montre la réalité et l'importance du multilinguisme. Politiques linguistiques et éducatives doivent tenir compte de la place des langues nationales, plusieurs types de réaction des systèmes éducatifs étant possibles face à la diversité linguistique. Les études de cas qui suivent l'analyse des discours et des pratiques illustrent cette situation.

PILHION Roger coord., Une politique pour le français, Paris, France, ministère des Affaires étrangères, La Documentation française, 1996, 124 p.

Cet ouvrage collectif présente les missions et les actions conduites dans le domaine de la promotion de la langue française par le réseau culturel et linguistique français du ministère des Affaires étrangères.

POCHE Bernard, Les langues minoritaires en Europe, Grenoble, France, PUG (Transeurope), 2000, $191 \mathrm{p}$.

Dans le cadre des polémiques qu'a suscitées la Charte européenne des langues régionales ou minoritaires et après quelques considérations sur «langue et société», l'auteur se livre ici à une analyse socio-historique de la constitution et de la pratique des diverses formes et des divers niveaux de langage.

POIGNANT Bernard, Langues et cultures régionales, juillet 1998, 39 p.

La mission confiée à l'auteur de ce rapport était «de faire un bilan exhaustif et objectif de l'enseignement [des langues régionales] et de faire toutes propositions sur l'évolution du dispositif». Disponible sur: http:/www.doc.française.fr

\footnotetext{
POIGNANT Bernard, Langues de France: osez l'Europe!, Montpellier/France, Indigène éditions, 2000, 159 p.

Agrégé d'histoire, Bernard Poignant est l'auteur du rapport à Lionel Jospin sur les langues et les cultures régionales. Dans le présent ouvrage, il explique pourquoi en France, pays de l'Étatnation, la question des langues régionales et minoritaires est si polémique. Il positionne la France par rapport à son engagement européen, traite de l'exception historique française et relit les grands auteurs qui ont pensé l'Etat, la nation et le peuple.
} 
REGNAULT Elizabeth dir., FOLLIET Henri dir., GAUTHIER Pierre-Louis dir., AFEC: Association francophone d'éducation comparée, Les enjeux du pluralisme linguistique pour les systèmes d'éducation et de formation, Education comparée, vol. 54, mai 2001, 244 p.

Le congrès de l'AFEC, qui a eu lieu en 1999 à Strasbourg et dont les actes sont publiés dans ce numéro, avait pour objectif d'étudier, dans une perspective comparatiste tant géographique qu'historique, la pluralité des langues à partir de situations de fait: coexistence de langues nationales, internationales, régionales, des migrants et autres minorités, mais aussi les contacts et les conflits linguistiques sur un territoire.

SAINT ROBERT Marie-Josée de, La politique de la langue française, Paris, PUF (Que sais-je ?), 2000, 127 p., bibliogr.

Après un état des lieux des différentes représentations de la langue française et des fondements de l'intervention de l'Etat, sont analysées les grandes étapes de la politique linguistique menée par la Ve République depuis 1960. Le lecteur découvre comment une volonté politique se traduit en action et comment ont été gérées les forces contradictoires en présence.

SANTAMARIA Antonio, BEN ACHOUR Yadh, BUGARSKI Ranko, SKILJAN Dubravko, SEBAA Rabeh, TSERONIS Assimakis, PAPPE Ilan, SUMANJSKI Berislav, CASULE Ilija, VLAISAVLJEVIC Ugo, "Politiques de la langue», Transeuropéennes, $n^{\circ}$ 14-15, mars 1999, p. 33-141.

A la suite d'un atelier organisé en Macédoine (Ohrid, mai 1998) intitulé «Politiques de la langue dans les sociétés multiculturelles», la revue Transeuropéennes présente un dossier (bilingue anglais-français) sur l'examen des politiques de la langue et leurs implications. Les différents auteurs, à travers l'exemple de la Catalogne, des pays du Maghreb, d'Israël, de la Grèce et de la zone des Balkans, reviennent sur les enjeux politiques de la langue qui, «dans l'élaboration ou la destruction d'un espace public sont cruciaux».

SIGUAN Miquel, L'Europe des langues, Liège/Belgique, Mardaga (Psychologie et sciences humaines), 1996, 200 p.

Les choix politiques et éducatifs de l'Europe ne peuvent se faire sans ignorer les origines historiques de la pluralité linguistique européenne. L'auteur les expose ici en soulignant les relations étroites et complexes entre langue et identité nationale. Il décrit les politiques linguistiques adoptées par les différents États pour faire face à leur propre diversité linguistique. Il analyse les comportements langagiers dans les sociétés multilingues et la présence croissante de l'anglais dans de nombreux domaines. Il aborde enfin les problèmes de l'enseignement des langues et de l'éducation bilingue. Son ouvrage, écrit avec une grande clarté et guidé par l'ambition avouée de tracer les voies d'une politique linguistique pour l'Union européenne, propose de viser à un équilibre entre la fidélité à sa langue et la convivialité avec les autres.

VIGNER Gérard coord., "Promotion, réforme des langues et systèmes éducatifs", Etudes de linguistique appliquée, $n^{\circ} 103$, juillet 1996, p. 261-384.

Deux grands thèmes pour ce numéro autour des politiques linguistiques dans le monde. Dans une première partie sont rassemblés des articles traitant de l'offre et des enjeux linguistiques: coopération transfrontalière franco-allemande, multiplication de l'offre dans une académie, bilan du programme Lingua au moment où il est intégré dans un programme plus large (Socrates) et situation de l'Amérique latine au regard de l'enseignement des langues. Dans la seconde partie on traite des politiques d'appui aux langues émergentes, c'est-à-dire entrant pour la première fois dans un système éducatif. C'est le cas du Pacifique Sud francophone (NouvelleCalédonie, Vanuatu, Wallis et Futuna), du Sénégal dans les années 70, des Seychelles. Le numéro comprend également un article sur l'Afrique en général et une synthèse de Gérard Vigner. 


\section{Études de la Commission européenne}

Les sites principaux à consulter

http://europa.eu.int/comm/education/languages

http://europa.eu.int/comm/education/languages/consult/action_fr.pdf

http://europa.eu.int/comm/education/policies/lang/languages/index_fr.html

http://www.eurydice.org

BLONDIN Christiane coord., Commission européenne. DG XXII: Education Formation Jeunesse, L'apprentissage des langues vivantes en milieu scolaire dans l'Union européenne, Bruxelles, EUR-OP (Etudes), 1997, 153 p., bibliogr.

A la suite du livre blanc «Enseigner et apprendre: vers la société cognitive», cette synthèse des résultats de la recherche en ce domaine se veut un outil de référence pour l'enseignement des langues dans l'Union européenne.

COMMISSION EUROPEENNE. Eurostat: office statistique de Bruxelles, Eurydice: Réseau européen d'information sur l'éducation en Europe, Les chiffres clés de l'éducation en Europe 2002: langues étrangères, Luxembourg, Office des publications officielles des Communautés européennes (Les chiffres clés de l'éducation en Europe), 2002, 27 p.

Cet opuscule sur les langues étrangères en Europe constitue l'un des chapitres de l'ouvrage sur les chiffres clés de l'Education édité par la Commission européenne. Il rassemble de nombreux tableaux statistiques sur l'organisation et l'enseignement des langues étrangères dans trente pays d'Europe.

COMMISSION EUROPEENNE. DG XXII: Education Culture. Formation professionnelle. Politique des langues, Enseignement et apprentissage des langues étrangères: action communautaire, 2001, 11 p. téléchargées du site:

http://europa.eu.int/comm/education/languages/consult/action_fr.pdf

Par ce document, la Commission a voulu faire le point sur les implications de la Communauté européenne dans le domaine de l'apprentissage des langues étrangères et dresse un panorama des principales innovations et des actions entreprises au rang desquelles les programmes Lingua, Socrates et Leonardo da Vinci. Ce texte rappelle également les actions de l'Union en faveur des langues régionales et minoritaires et pour un apprentissage précoce. Un tableau des différents sites internet de la Commission sur les langues clôt le document.

COMMISSION EUROPEENNE, Promouvoir l'apprentissage des langues et la diversité linguistique: consultation. Document de travail des services de la Commission, novembre 2002, 24 p. téléchargées du site: http://europa.eu.int/comm/education/languages/consult/consult_fr.pdf

Suite à l'Année européenne des langues, la Commission a décidé d'envisager de nouveaux moyens et méthodes pour pomouvoir l'apprentissage des langues. Le présent document invite les parties prenantes à donner leur avis sur la forme que devrait prendre cette action concertée; après avoir rappelé quelques grands principes, il émet des propositions, notamment pour un meilleur enseignement des langues à l'école (formation des enseignants, matériel et équipement) et formule les questions correspondantes. 


\section{EURYDICE, La place des langues étrangères dans les systèmes éducatifs euro-} péens 1999/2000, Bruxelles (Eurydice Focus), 2000, 44 p.

En préliminaire à l'Année européenne des langues (2001), Eurydice présente l'organisation de l'enseignement scolaire des langues étrangères dans vingt-neuf pays européens à l'aide d'une série de diagrammes représentant la place qu'elles occupent tout au long du parcours scolaire. Ces diagrammes illustrent, entre autres, l'âge auquel débute cet enseignement, le nombre de langues enseignées, leur caractère obligatoire ou optionnel etc., au niveau de l'enseignement pré-élémentaire, élémentaire et secondaire.

EURYDICE: le réseau d'information sur l'éducation en Europe, L'enseignement des langues étrangères en milieu scolaire en Europe, Bruxelles, Eurydice, 2000, $372 p$.

Étude comparative sur l'enseignement des langues étrangères dans les vingt-neuf pays participant au programme Socrates, qui offre une analyse détaillée de l'organisation pédagogique de cet enseignement, des approches et du contenu des programmes d'études, des mesures prises dans la formation et le recrutement des enseignants ainsi que des actions de soutien linguistique développées dans les systèmes éducatifs à l'égard des langues minoritaires. Cette étude apporte également une perspective historique des nombreuses réformes menées dans ce domaine depuis plusieurs décennies.

EURYDICE: Réseau européen d'information sur l'éducation en Europe, Enseignement des langues: bibliographie thématique, Bruxelles, Commission européenne. Direction générale de l'éducation et de la culture (Bibliographie thématique), 2001, 38 p.

Dans cette bibliographie, publications, rapports internationaux et sites internet sont recensés pour la plupart depuis 1995. Les références s'organisent en huit catégories ayant trait au domaine de l'enseignement des langues en Europe. Au sein de chaque chapitre, elles sont présentées chronologiquement, accompagnées d'un résumé et de mots clés.

INRA (EUROPE): International Research Associates - European Coordination Office, Les Européens et les langues: rapport, Bruxelles, INRA, février 2001, 55 p. + annexes téléchargées du site http://europa.eu.int

Un sondage spécial Eurobaromètre a été réalisé dans l'ensemble des États membres de l'Union européenne en décembre 2000 à la demande de la direction générale de l'Éducation et de la Culture (unité Politique des langues) de la Commission européenne. Au total, plus de 15900 personnes ont donné leur avis et fait part de leur expérience dans le domaine des langues. Le présent rapport analyse la connaissance des langues sous différents aspects: langues connues et langues utiles à connaître, pratique des langues étrangères, modalités d'apprentissage, motivations et opinions sur la connaissance et l'apprentissage des langues. Le questionnaire en anglais et en français figure en annexe. Le rapport est consultable en format PDF http://europa.eu.int/comm/public_opinion/archives/eb/ebs_147_fr.pdf.

PILOS Spyridon, "L'étude des langues étrangères dans les systèmes scolaires d'Europe», Luxembourg, Eurostat, Statistiques en bref: population et conditions sociales, $n^{\circ} 3$, avril 2001, 8 p.

Ce document statistique présente la situation de l'étude des langues étrangères au sein des sytèmes éducatifs formels de l'Union européenne, dans l'enseignement primaire et secondaire; il ne traite pas de l'étude des langues dans l'enseignement supérieur, ni des langues mortes et les indicateurs présentés ici concernent l'étude des langues étrangères en tant que matières à part entière et non comme langues d'enseignement pour d'autres disciplines. 


\section{Études du Conseil de l'Europe}

Les sites principaux à consulter:

http://www.coe.int/portalT.asp

site de Graz: http://www.ecml.at/

BEACCO Jean-Claude, BYRAM Michael, Guide pour l'élaboration des politiques linguistiques éducatives en Europe: de la diversité linguistique à l'éducation plurilingue: version intégrale, projet 1 révisé, Strasbourg, Conseil de l'Europe, division des Politiques linguistiques, avril 2003, 115 p.

La finalité de ce guide est de proposer une analyse qui serve de cadre à l'élaboration des enseignements de langues dans les États membres ainsi qu'une réflexion sur les politiques linguistiques européennes. La première partie présente une vision des caractéristiques communes des politiques linguistiques actuellement menées dans les États membres et aboutit à une interprétation différente des principes de diversité linguistique et de plurilinguisme tels qu'ils sont appliqués à travers les politiques linguistiques de chaque État. La deuxième partie décrit une démarche de gestion et de mise en œuvre des politiques linguistiques dans une perspective méthodologique. Enfin, la dernière partie propose un inventaire des moyens techniques permettant d'organiser des enseignements en langues correspondant aux principes évoqués dans la première partie. Ce guide est soumis à consultation qui conduira à la mise au point d'une version révisée; il est consultable sur www.coe.int

BYRAM Michael, ZARATE Geneviève, NEUNER Gerhard, La compétence socioculturelle dans l'apprentissage et l'enseignement des langues, Strasbourg, Conseil de l'Europe (Education), 1998 (réimp. de 1997), 124 p., bibliogr.

Cet ouvrage est la reprise de deux études datant de 1994: Définitions, objectifs et évaluation de la compétence socioculturelle (Byram, Zarate) et Le Rôle de la compétence socioculturelle dans l'enseignement et l'apprentissage des langues vivantes (Neuner), cette dernière étant disponible en anglais.

CONSEIL DE L'EUROPE, La diversité linguistique en faveur de la citoyenneté démocratique en Europe: vers un document-cadre pour des politiques linguistiques éducatives, Strasbourg, Conseil de l'Europe, 2001, 185 p.

Cet ouvrage est fondé sur les conclusions d'une conférence portant sur le rapprochement des peuples d'Europe à travers l'apprentissage des langues. Rapports, discours et discussions défendent, pour la plupart, l'idée que la diversité linguistique est en mesure de jouer un rôle éminent dans la promotion de la citoyenneté démocratique; ils apportent aussi des suggestions en matière de politiques de promotion de la diversité linguistique.

CONSEIL DE L'EUROPE, Division des langues vivantes, Centre européen pour les langues vivantes (CELV), Graz/Autriche, Identité sociale et dimension européenne: la compétence interculturelle par l'apprentissage des langues vivantes, Strasbourg, Conseil de l'Europe, 2001, 198 p.

Présentation du projet du Conseil de l'Europe et interventions des participants sur l'identité sociale et l'enseignement des langues étrangères puis sur la dimension européenne. Les deuxième et troisième parties rendent compte, par pays, des modules pédagogiques et des modules de formation de formateur. 
CONSEIL DE L'EUROPE. Conseil de la coopération culturelle. Comité de l'Education.Division des langues vivantes, Cadre européen commun de référence pour les langues : apprendre enseigner évaluer, Paris, Didier, 2001, 191 p., bibliogr., annexes.

Résultat d'une recherche menée pendant plus de dix ans par des linguistes de renom dans les quarante-et-un États membres du Conseil de l'Europe, ce document vise à encourager les praticiens des langues vivantes à questionner leur façon d'enseigner et à faciliter les échanges d'informations entre praticiens et apprenants. Les chapitres traitent successivement des principes fondamentaux et de leurs conséquences pratiques, des niveaux communs de référence, de l'utilisation de la langue, des compétences de l'apprenant/utilisateur, des opérations d'enseignement et d'apprentissage des langues, de diversification linguistique dans la conception de curriculum et d'évaluation.

CONSEIL DE L'EUROPE. Division des politiques linguistiques, Common European Framework of Reference for Languages: Learning Teaching Assessment. Case studies, éditions du Conseil de l'Europe (Politiques linguistiques), 2002, 198 p.

Ce document analyse la façon dont le Cadre européen commun de référence est utilisé pour promouvoir l'enseignement des langues étrangères dans des pays d'Europe comme la Pologne, la Catalogne, la Finlande, l'Irlande et la Suisse. Il développe dans les chapitres suivants l'utilité du Cadre européen pour mesurer les compétences langagières, notamment pour les adultes appenants et pour l'entrée à l'université. Dans le domaine des certifications, les travaux de l'association ALTE (Association of Language Testers in Europe) sont rappelés en fin d'ouvrage.

CONSEIL DE L'EUROPE. Portfolio européen des langues,

Portfolio européen des langues: pour jeunes et adultes, Caen, CRDP, Paris, Didier, janvier 2001, 36 p.

Mon premier Portfolio des langues, CIEP, Paris, Didier, 2001.

Portfolio européen des langues: collège, CIEP, ENS-Lyon, à paraître aux éditions Didier, septembre 2003.

Le porfolio est commun aux différents pays européens dans sa structure et par les références à la même échelle de compétences. Ce document s'articule autour de trois parties: le passeport de langues, où figure le niveau de performance en compréhension et expression - la bibliographie langagière, pour que l'apprenant fasse le point sur son niveau, se fixe des objectifs et auto-évalue ses progrès - le dossier, qui comportera les documents attestant d'un niveau de performance, tels que les travaux réalisés ou les diplômes.

FINK Bärbel ed., European Center for Modern Languages, Graz, Autriche, Commission Européenne. DG XXII, Modern language learning and teaching in central and eastern Europe: which diversification and how can it be achieved ?, Strasbourg, Conseil de l'Europe, 1998, 369 p.

Tables rondes et ateliers rendent compte de l'actualité la plus récente de la situation de l'enseignement des langues dans cette large partie de l'Europe aussi bien du point de vue des données chiffrées que du point de vue didactique. On peut également accéder à des exposés pour des pays particuliers, ce qui complète utilement les rapports nationaux d'Euridyce présents sur le site http://culture.coe.fr/ecml/publ 
GOGOLIN Ingrid, Diversité linguistique et nouvelles minorités en Europe, Strasbourg, Conseil de l'Europe (Guide pour l'élaboration des politiques linguistiques éducatives - De la diversité linguistique à l'éducaton plurilingue), 2002, 22 p., bibliogr.

Le premier chapitre de ce document illustre la diversité linguistique contemporaine en Europe. Le second chapitre aborde le rôle des langues pour les nouvelles minorités. Le troisième chapitre présente un modèle pour la construction d'une politique d'éducation linguistique ayant pour but de réspecter et d'enscourager la diversité.

GRIN François, Conseil de l'Europe. Division des politiques linguistiques. DG IV/Strasbourg, L'économie de la langue et de l'éducation dans la politique d'enseignement des langues: étude de référence, Strasbourg, Conseil de l'Europe. Division des Politiques linguistiques (guide pour l'élaboration des politiques linguistiques éducatives en Europe - de la diversité linguistique à l'éducation plurilingue), octobre 2002, 49 p., bibliogr.

Ce document met en évidence la complexité des questions d'enseignement des langues. Il décrit les démarches et les outils conceptuels permettant d'analyser les contextes éducatifs sous l'angle des langues et d'organiser les formations en tenant compte des principes adoptés par le Conseil de l'Europe. Celui-ci travaille à valoriser le plurilinguisme et à le mettre au cœur des politiques linguistiques éducatives. Ces dernières sont analysées dans une perspective économique, notamment en termes de valeur de la langue, coûts de l'investissement en capital humain et estimation des taux de rendement privés des compétences en langues secondes.

MILANOVIC M., Conseil de l'Europe. Division des politiques linguistiques, Cadre européen commun de référence pour les langues: apprendre enseigner évaluer. Evaluation de compétences en langues et conception de test, Strasbourg, éditions du Conseil de l'Europe (Politiques linguistiques), octobre 2002, 52 p.

Ce guide se veut un outil pour tous ceux qui se sont engagés dans l'élaboration de tests de langue en prenant comme base de travail le Cadre européen commun de référence pour les langues: Apprendre, Enseigner, Évaluer. Il peut être utile aussi bien en situation officielle d'examens que dans les pratiques de classe.

PIRI Ritta, Conseil de l'Europe. Division des politiques linguistiques. DG IV/Strasbourg, L'enseignement des langues de moindre diffusion comme langues étrangères dans d'autres pays: guide pour l'élaboration des politiques linguistiques éducatives en Europe - De la diversité linguistique à l'éducation plurilingue, Strasbourg, Conseil de l'Europe, 2002, 32 p.

Ce document aborde un aspect spécifique de la problématique des politiques linguistiques éducatives en Europe, à savoir la question des "petites langues ou langues mineures». Il se dégage de cette étude que les principes généraux de l'enseignement et de l'apprentissage des langues s'appliquent également aux langues de moindre diffusion mais qu'il est nécessaire de s'assurer de l'existence d'une volonté politique favorable à ce dernier enseignement. L'auteur, développant cette idée, indique les instruments de pilotage pour les politiques et donne un exemple de schéma des éléments clés du développement d'un programme d'enseignement des langues. 
SKUTNABB-KANGAS Tove, Pourquoi préserver et favoriser la diversité linguistique en Europe? Quelques arguments, Strasbourg, Conseil de l'Europe, 2002, 22 p., bibliogr.

L'introduction présente un état des lieux de la diversité linguistique en Europe, continent le plus pauvre sur le plan linguistique. L'auteur clarifie ensuite les concepts et les définitions, puis argumente en faveur de la diversité linguistique (arguments historiques et économiques).

TRIM John, Apprentissage des langues et citoyenneté européenne: rapport final du groupe de projet (activités 1989-1996), Strasbourg, Conseil de l'Europe, 1997, 105 p., bibliogr.

Ce document est une synthèse des activités du Conseil de l'Europe dans le domaine des langues pour la période étudiée et un exposé des travaux en cours. Il dresse l'historique du projet « Apprentissage des langues et citoyenneté européenne », expose ses buts, présente les ateliers qui ont eu lieu, le projet de Cadre européen de référence pour l'enseignement/apprentissage des langues, les aides au développement de l'enseignement des langues dans les nouveaux Etats membres, les développements connexes (Centre européen des langues par exemple). Il pose également la question de la diffusion de l'information sur ces travaux. En annexe, les développements intervenus dans les États membres, la liste des ateliers et programmes d'action tenus ainsi que les publications et documents édités au cours de cette période.

\section{Études consacrées à L'Année européenne des langues 2001}

http://www.ecml.at/eyl/

BUNJES Ulrich, Conseil de l'Europe. Division des politiques linguistiques. DG IV, Année européenne des langues 2001: évaluation finale. Rapport, Strasbourg, Conseil de l'Europe, mai 2002, 55 p.

Ce rapport synthétise les informations livrées par les quarante-et-un coordinateurs nationaux, à partir d'un questionnaire, à la fin de l'Année européenne des langues (AEL) 2001. Après avoir décrit l'organisation de l'AEL, il donne une évaluation de son impact, des exemples de bonne pratique et le suivi de cette manifestation. Parmi les annexes: questionnaire d'évaluation, thèmes proposés, matériel de promotion produit par le Conseil de l'Europe, budget.

COMMISSION EUROPEENNE, Mise en ouvre et résultats de l'année européenne des langues 2001: rapport de la Commission au Conseil, au Parlement européen, au Comité économique et social et au Comité des régions, novembre 2001, 41 p. téléchargées du site:

http://europa.eu.int/comm/education/languages/download/rap_fr.pdf

Bilan et suites de l'Année européenne des langues. 
GAUDEMAR Jean-Paul de dir., LANG Jack, COSTE Daniel, BEACCO JeanClaude, CANDELIER Michel, ZARATE Geneviève, BRENNAN Paul, JOUTARD Philippe, MONNANTEUIL François, PHILIPPS Joseph, MACCARIO Bernard, GOULLIER Francis, DESCO: Direction de l'enseignement scolaire, Paris, L'enseignement des langues vivantes perspectives: Actes du séminaire des 27 et 28 mars 2001 - lycée Henry IV et École normale supérieure rue d'Ulm à Paris, Versailles, CRDP de l'Académie de Versailles, 2001, 123 p.

En mars 2001, durant l'Année européenne des langues, le ministère de l'Éducation nationale a organisé un séminaire national qui a réuni tous les acteurs de l'enseignement des langues vivantes, des inspecteurs généraux de langues aux directeurs d'IUFM, ainsi que les représentants des ambassades des cinq pays européens dont les langues sont les plus enseignées en France. Interventions et ateliers ont permis d'examiner, sur l'ensemble de la scolarité des élèves, la cohérence et la continuité des approches pédagogiques, la diversité des parcours et l'ouverture des enseignements à la dimension internationale.

MIS Bernard coord., L'enseignement des langues vivantes en Europe: le défi de la diversification, Paris, Didier (Les cahiers du CIEP), 2002, 128 p.

Ce numéro des Cahiers $d u$ CIEP rend compte des travaux tenus à Paris au cours de la Journée européenne des langues, le 26 septembre 2001. Outre les comptes rendus des tables rondes et des ateliers de cette journée de réflexion, on y trouve les témoignages de responsables de politique linguistique de quinze pays et trois articles sur diverses intiatives françaises en matière de promotion des langues étrangères. Les textes de référence sur le plurilinguisme sont donnés en annexe.

PUREN Christian, BREUGNOT Jacqueline, VILLANUEVA Marisa Luisa, FRATH Pierre, "L'Europe des langues: dossier», Langues modernes (Les), $n^{\circ} 3$, septembre 2001, p. 3-39.

Dans le cadre de l'Année européenne des langues, la revue les Langues modernes a tenu à consacrer un numéro spécial sur l'Europe et les langues. Après un article introductif qui détaille le contenu des principaux programmes de l'Union européenne en matière d'apprentissage des langues, on trouve un exposé comparatif entre le système scolaire français et le système allemand et les conséquences qui en découlent pour l'enseigement des langues. Une autre contribution étudie l'enseignement des langues en Espagne. Le dernier article de ce dossier tente d'expliquer les avantages et les dangers de l'hégémonie actuelle de l'anglais. 